Article

\title{
Optimal Placement of PMU to Enhance Supervised Learning-Based Pseudo-Measurement Modelling Accuracy in Distribution Network
}

\author{
Kyung-Yong Lee ${ }^{1}\left(\mathbb{D}\right.$, Jung-Sung Park ${ }^{2}(\mathbb{D})$ and Yun-Su Kim ${ }^{1, *(D)}$ \\ 1 Graduate School of Energy Convergence, Gwangju Institute of Science and Technology, \\ Gwangju 61005, Korea; leeky0807@gm.gist.ac.kr \\ 2 KEPCO Research Institute, 105, Munji-Ro, Yuseong-Gu, Daejeon 34056, Korea; jindulfa@kepco.co.kr \\ * Correspondence: yunsukim@gist.ac.kr
}

Citation: Lee, K.-Y.; Park, L.-S.; Kim, Y.-S. Optimal Placement of PMU to Enhance Supervised Learning-Based Pseudo-Measurement Modelling Accuracy in Distribution Network. Energies 2021, 14, 7767. https:// doi.org/10.3390/en14227767

Academic Editor: Nikolaos Manousakis

Received: 13 October 2021 Accepted: 17 November 2021 Published: 19 November 2021

Publisher's Note: MDPI stays neutral with regard to jurisdictional claims in published maps and institutional affiliations.

Copyright: (c) 2021 by the authors. Licensee MDPI, Basel, Switzerland. This article is an open access article distributed under the terms and conditions of the Creative Commons Attribution (CC BY) license (https:// creativecommons.org/licenses/by/ $4.0 /)$.

\begin{abstract}
This paper introduces a framework for optimal placement (OP) of phasor measurement units (PMUs) using metaheuristic algorithms in a distribution network. The voltage magnitude and phase angle obtained from PMUs were selected as the input variables for supervised learningbased pseudo-measurement modeling that outputs the voltage magnitude and phase angle of the unmeasured buses. For three, four, and five PMU installations, the metaheuristic algorithms explored 2000 combinations, corresponding to $40.32 \%, 5.56 \%$, and $0.99 \%$ of all placement combinations in the 33 -bus system and $3.99 \%, 0.25 \%$, and $0.02 \%$ in the 69 -bus system, respectively. Two metaheuristic algorithms, a genetic algorithm and particle swarm optimization, were applied; the results of the techniques were compared to random search and brute-force algorithms. Subsequently, the effects of pseudo-measurements based on optimal PMU placement were verified by state estimation. The state estimation results were compared among the pseudo-measurements generated by the optimal PMU placement, worst PMU placement, and load profile (LP). State estimation results based on OP were superior to those of LP-based pseudo-measurements. However, when pseudo-measurements based on the worst placement were used as state variables, the results were inferior to those obtained using the LP.
\end{abstract}

Keywords: metaheuristic algorithms; optimal placement; phasor measurement units (PMU); pseudo-measurement; state estimation

\section{Introduction}

Energy conversion is increasingly drawing attention due to the goals of the Kyoto Protocol and Paris Climate Agreement. Renewable energy sources are steadily being incorporated into distribution systems, and the systems are changing accordingly. However, with this transition, distribution systems can suffer problems such as voltage instability, thermal overload, and monitor distribution systems to ensure stable operation. However, meters installed in power distribution systems are often limited to substations or lowvoltage networks [1] due to the high cost of installing phasor measurement unit (PMU) necessary to make the most of a limited number of meters.

Traditionally, supervisory control and data acquisition (SCADA) systems have been used to observe the state of the system. A common task SCADA performs is state estimation that relies on unsynchronized and slow measurements [2]. Measurements based on SCADA have some limitations and errors, such as slow duty cycle and measurement delay due to communication bias. To solve these problems and limitations, wide-area monitoring protection and control (WAMPAC) has been proposed [3,4]. The PMU is a key component of WAMPAC, facilitating real-time calculations and synchronized phasor measurements of voltages and currents in the power grid. PMUs can utilize global positioning systems (GPS) to achieve precision and accuracy, so their use is rapidly increasing to improve monitoring 
of power grids. However, since PMUs are expensive equipment, installing them in all locations would require an astronomical cost. In order to solve this problem, studies have been conducted to optimize the number and installation location of PMUs to secure system observability at the lowest cost.

There are numerous studies of optimal meter placement using heuristic algorithms in power systems. The authors of [5] proposed heuristic search-based solutions to minimize the cost of installing meters by accepting a trade-off between estimation accuracy and meter installation costs. In [6], a heuristic meter placement for load estimation is presented; this method can handle large distribution systems with only moderate computation requirements, making it easier to handle the actual constraints of meter failure backups, space availability, existing meters, and unbalanced distribution systems. Additionally, their approach [6] provides a reasonable compromise in terms of the complexity of meter deployment for distribution planning and operation. In [7], an ordinal optimization technique was introduced in an attempt to improve the estimation accuracy. According to the author, the simplicity and computational efficiency of this algorithm are much improved over other discovery-based search algorithms, making it suitable for practical purposes. In [8], genetic algorithm (GA)-based solutions are provided to improve estimation accuracy. A number of studies have been conducted on the optimal placement (OP) of meters, with the intent of securing the observability of the system and thus increasing the estimation accuracy. In [9], a GA technique is used to determine optimal meter placement, in which the optimal meter layout was identified for maintaining the system's observability. In [10], a GA was used for optimal meter placement, for static estimation of harmonic sources in the power system. GA techniques tend to show similar results to complete enumeration techniques. One of the conclusions of this study was that the superiority of GA techniques over complete enumeration increases as the number of meters or the system size used for placement increases. In [11], binary particle swarm optimization (BPSO) techniques were used to optimally position PMUs. BPSO-based approaches have been used to investigate PMU placement with two main objectives: minimizing the number of PMUs required to maintain full observability of the system and maximize measurement redundancy for all buses in the system.

Numerous studies have been conducted on the placement of meters to secure observability or improve state estimation accuracy. However, the optimal meter placement for this purpose has constraints that require minimal meter guarantees. If it is impossible to achieve observability of the power system with only the installed meters, pseudo-measurements can be used. The basic method of generating pseudo-measurements is to use historical data from feeders and load profiles (LPs) [12]. However, pseudo-measurements generated in this way tend to have poor accuracy and high variance in state estimations [13]. Numerous studies have obtained more accurate pseudo-measurements using artificial intelligence (AI) techniques [13-20]. In [14], optimal meter placement was achieved by estimating the voltage data via AI techniques; with their method, it is possible to quickly select the optimal meter position based on simple rules, with only the voltage magnitude estimates taken into account. The authors in [13] proposed an artificial neural network (ANN)-based pseudo-measurement technique to estimate the active/reactive power of the line for state estimation in the distribution system. The proposed technique generated two ANN models that trained LPs and offline load flows, or historical data, to determine the active/reactive injection power.

In [15], linear regression (LR), support vector machine, and feed-forward neural network techniques were used to estimate the voltage at the measured point, and the voltage and phase angle at the unmeasured point, for comparison of the results. Modeling with AI models tends to be more accurate than that based on LPs. It also enables better state estimation with fewer meters than is required to achieve observability or improve estimation accuracy. However, the basis for selecting the input bus was not considered. In [16], pseudo-measurements were modeled using ANN and a Gaussian mixture model (GMM) in a low-voltage distribution system; the GMM was used to determine the variance of 
the pseudo-measurements generated by the ANN. In [17], the author estimated pseudomeasurements using gradient-boosting tree techniques; this approach requires extensive training time, but can be performed offline as it uses only historical data. The trained model provides fast predictions. The main advantage is that the model is learned based on user-level measurement data, unlike the LP-based model, and generates more accurate pseudo-measurements. In addition, the model is built step-by-step, so it can be applied to distribution systems with frequent topology changes. Depending on the topology, a subset can be used to generate pseudo-measurements. In [18], ANN and Fourier decomposition were used to model the pseudo-measurements' typical active power/reactive power and the standard deviation profile. In [19], an extreme learning machine-based pseudo-measurement modeling technique is proposed that uses injection power measured by the supervisory control and data acquisition as input, and real and imaginary parts of the bus voltage as output. This technique not only improves the accuracy of the state estimation, but also significantly reduces the computational time. In [20], a convolutional neural network-based method for pseudo-measurement modeling in a distribution system state estimation was studied. This method improves the efficiency of the calculations. In many cases, pseudo-measurement modeling using AI techniques requires model generation through learning data. The meter's position influences AI-based pseudo-measurement modeling, as data acquisition in the power system is primarily done by meters. However, as the number of nodes in the target system increases, or as the number of meters installed reaches half of the total number of nodes, the number of nodes required for exhaustive exploration also increases. Therefore, the study is needed to derive optimal meter placement through efficient exploration, for AI-based pseudo-measurement modeling.

In order to calculate voltage magnitude and phase angle of the buses by the power flow calculation, at least two parameters among active/reactive power, voltage magnitude, and phase angle of all buses are required. As obtaining sufficient parameter information needed for power flow calculation of all buses in a system lacking meters is impossible, power flow calculation cannot be used to calculate the parameters of unmeasured points. Even if the same unmeasured point is targeted, a different LR model is generated as the measured point is changed. It is difficult to determine which model is generated according to the measurement point because there are insufficient data to explain the relationship between the measurement point and the unmeasured point. Accordingly, approaches based on metaheuristics can find good solutions in less computational time, even for uncertain and highly complex problems like PMU placement for supervised learning-based pseudomeasurement modeling [21].

Generating supervised learning models requires sufficient data to be used for learning and validation. In this study, the active/reactive power injected into each node was randomly generated with Gaussian noise $(\sigma=25 \%)$, and power flow calculations were used to obtain voltage and phase angle data. Power flow calculations were implemented by pandapower, a Python open source library.

$$
\begin{aligned}
& P_{i}=\sum_{k=1}^{n}\left|V_{i} \| V_{k}\right|\left(G_{i k} \cos \theta_{i k}+B_{i k} \sin \theta_{i k}\right), \quad i=1,2, \cdots, n \\
& Q_{i}=\sum_{k=1}^{n}\left|V_{i} \| V_{k}\right|\left(G_{i k} \sin \theta_{i k}-B_{i k} \cos \theta_{i k}\right), \quad i=1,2, \cdots, n
\end{aligned}
$$

where $P_{i}, Q_{i}, G_{i k}, B_{i k}, V_{i}$, and $V_{k}$ are the active and reactive power of the $i$ th bus of the network, the elements of the conductance matrix and susceptance matrix, and the voltages of the $i$ th bus and $k$ th bus, respectively. Equations (1) and (2) were used to generate data for supervised learning model training. The details of this process can be found in [15].

In this paper, two metaheuristic algorithms, GA and PSO, which have the most related papers among metaheuristic techniques on Google Scholar, were used [22]. Optimal meter placement results were compared with random search (RS) [23] and brute force (BF) [24] 
techniques. The estimated pseudo-measurements based on OP were compared to pseudomeasurements based on LPs, in which each pseudo-measurement was used as a state variable for state estimation. The criterion for the optimal position is the total vector error (TVE) of all unmeasured points estimated by LR techniques.

The main contributions of the proposed approach are: (i) Provides an approach for optimal PMU placement for supervised learning-based pseudo-measurement modeling techniques, which previous studies have not focused on. (ii) Even in highly complex systems, excellent placement can be found in a short time through effective search strategies of metaheuristic techniques. (iii) Various metaheuristic techniques can be applied using a proposed framework that is not limited to specific metaheuristic techniques. (iv) In [15], only the performance of the estimated pseudo-measurement value was evaluated, but in this paper, the weighted least squares state estimation was performed considering the uncertainty of the pseudo-measurement value and compared with the load profilebased method.

The rest of this paper consists of four sections. The pseudo-measurement modeling technique based on LR is described in Section 2. Problem formulation and the application of metaheuristic techniques for optimal meter placement is introduced in Section 3. Simulation results for comparison of optimal meter placement and the performance of the pseudomeasurements generated at that location are provided in Section 4 , and a summary and conclusion are provided in Section 5 .

\section{Pseudo-Measurement Modeling}

Here we used LR to generate the supervised learning models. LR is used by scipy, a Python open-source library. LR techniques show excellent pseudo-measurement estimation performance and are easily applied in large-scale systems using relatively simple computational processes [15]. The expression for LR is as followed (Equation (3)):

$$
\mathbf{y}=\mathbf{X} \boldsymbol{\beta}+\boldsymbol{\epsilon},
$$

where $\mathbf{y}$ is the dependent variable vector of dimension $u \times 1$. Our pseudo-measurement model comprised the voltage magnitude and phase angle of the estimation target buses, where $m$ is the number of buses being measured. The $\mathbf{X}$ value on the right side is the independent variable matrix of dimension $u \times(m+1)$, with the first column consisting of 1. Vector $\beta$ is the coefficient variable of dimension $(m+1) \times 1$ and $\epsilon$ and $\epsilon$ is an error term vector of dimension $u \times 1$, which denotes an error between the dependent and independent variables. The details for this process can be found in [15].

\section{Problem Formulation}

In this section, the problem of PMU placement through metaheuristic techniques is formalized. Metaheuristic techniques explore PMU placement based on the TVE of unmeasured buses estimated by the LR model. Different LR models are generated depending on PMU placement, and the generated LR model estimates the parameters of the unmeasured point based on the parameters of the measured point. Estimating the model that will be generated is difficult because it requires a formula that clearly identifies the relationship between the parameters of the measured and unmeasured points. Metaheuristic methods can provide solutions to these problems through their own search strategies, whereas mathematical optimization techniques, such as linear programming and quadratic programming, are difficult to apply.

The criteria for assessing the accuracy of measurements of a PMU are defined in [25]. The phasor value of a sine wave is represented by two values: the amplitude and the phase. TVE can be utilized as an indicator of a PMU's performance by considering these two values together. TVE is calculated by comparing theoretical values with phasors of measurements. The expression is as follows (Equation (4)): 


$$
\operatorname{TVE}(n)=\sqrt{\frac{\left(\hat{X}_{r}(n)-X_{r}(n)\right)^{2}+\left(\hat{X}_{i}(n)-X_{i}(n)\right)^{2}}{X_{r}(n)^{2}+X_{i}(n)^{2}}},
$$

where $\hat{X}_{r}(n), \hat{X}_{i}(n)$ are real and imaginary part value of phasor measured by PMU; $X_{r}(n)$, $X_{i}(n)$ are real and imaginary part value of theoretical phasor calculated by power flow calculation. If the phasor of the unmeasured bus No. $n$ estimated by LR is inputted, the TVE of the bus is outputted.

The placement information and number of PMUs determine the data used to learn LR models. The values estimated by the learned LR model consist of the voltage magnitude and angle. Both are combined by TVE, making them one value. In other words, optimal PMU placement requires a solution to the nonlinear optimization problem that minimizes the value of the objective function, which depends on the placement and number of PMUs. The mathematical expression is the following (Equations (5)-(8)):

$$
\begin{gathered}
\mathbf{b}_{i}\left(N_{m}\right)=\left[\text { PMUlocation } 1, \text { PMUlocation } 2, \cdots, \text { PMUlocation } N_{m}\right], \quad i=1,2, \cdots, N_{p} \\
f\left(\mathbf{b}_{i}\left(N_{m}\right)\right)=\left[V_{i e 1}, \theta_{i e 1}, V_{i e 2}, \theta_{i e 2}, \cdots, V_{i N_{u}}, \theta_{i N_{u}}\right]
\end{gathered}
$$

using Equation (4), TVEs of each unmeasured buses can be obtained from Equation (6). The metaheuristic algorithm adjusts $\mathbf{b}_{i}$ to reduce $\operatorname{avg} T V E_{i}$.

$$
\begin{gathered}
\mathbf{T}_{i}=\left[T V E_{i 1}, T V E_{i 2}, \cdots, T V E_{i N_{u}}\right], \\
\operatorname{avg} T V E_{i}=\frac{1}{N_{u}} \sum_{k=1}^{N_{u}} T V E_{i k},
\end{gathered}
$$

where $\mathbf{T}_{i}$ is a vector consists of TVEs of each unmeasured buses, and $\mathbf{b}_{i}$ is placement of PMUs, the vector consists of locations where PMUs are installed. $N_{m}$ is the number of buses PMU installed, $N_{p}$ is the total number of particles (populations), and $N_{u}$ is the number of unmeasured buses. In other words, a metaheuristic algorithm is used to find the best placement $\mathbf{b}_{i}$ that minimizes average TVE. Metaheuristic algorithms are performed with the goal of finding combinations with lower average TVE values as the number of iterations (generations) increases.

Figure 1 shows the overall conceptual diagram of optimal PMU placement framework using metaheuristic technique. Voltage magnitude and phase angle data obtained from placement derived by metaheuristic techniques are used as input data to LR model training. Subsequently, test sets are used to evaluate the TVE. This process is repeated until the termination condition is satisfied. In this paper, first, a vector with 100 random PMU placement combinations is generated. The value of the vector can be any bus number except for the slack bus, because it is assumed that the PMU is installed on the bus. After that, each vector goes to the supervised learning model creation process to generate a linear regression model that estimates the voltage and phase angle of a bus that is not installed based on the voltage and phase angle of the bus where the PMU is installed. This process is repeated until all 100 vectors have been performed. Then, the value of each vector is updated to find a vector with a lower average TVE value through the Eigen strategy of the metaheuristic algorithm. This corresponds to the crossover and mutation process in the case of GA, and update velocity in the case of PSO. This process is performed 20 times. After all 20 runs are completed, the optimal location is selected as the location corresponding to the vector with the lowest average TVE value among the vectors found while 100 vectors are updated 20 times. The linear regression model created based on this optimal position generates pseudo-measured values for unmeasured points, and is used as a state variable for state estimation along with the measured values at the point where the PMU is installed.

In Figure 1, the box corresponding to the 'Metaheuristic algorithm', any algorithm that outputs a vector containing the arrangement information of the PMU according to 
iterative execution can be used even if it is not GA or PSO. The box corresponding to the 'Supervised Learning Model' can be applied not only to linear regression techniques but to any regression technique that can estimate the parameters of the unmeasured point with the measurement value of the PMU.

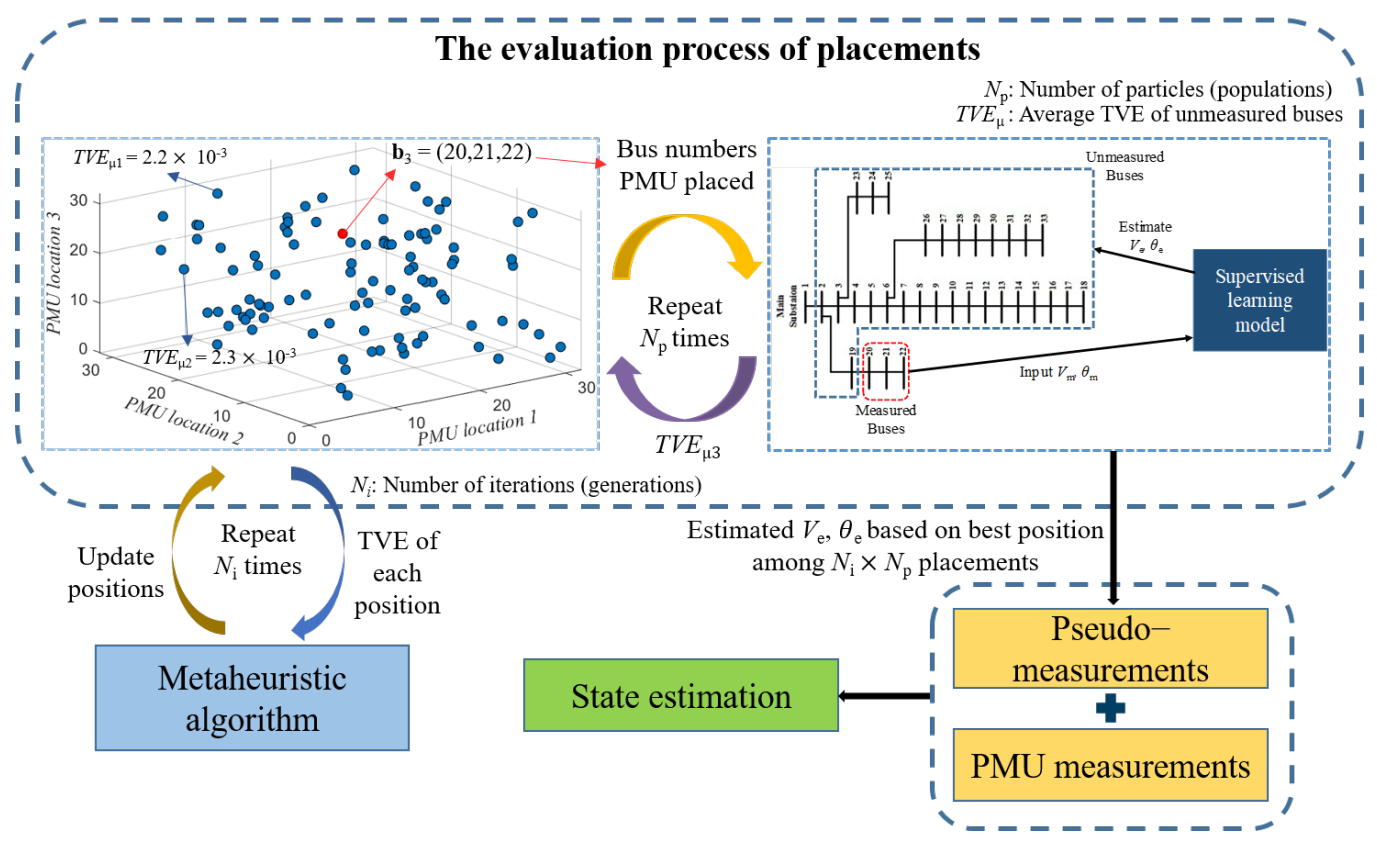

Figure 1. Overall conceptual diagram of the framework.

\subsection{Genetic Algorithms}

GA has been used in science and engineering as a computational model for adaptive algorithms and natural evolution systems to solve real-world problems [26]. By simulating the survival-of-the-deficit evolution strategy of chromosomes, the optimal combination of PMU placement is derived by crossover and mutation processes.

GA has a crossover process among multiple objects, this process enables efficient exploration compared to simple random exploration. Furthermore, the mutation process can improve the phenomenon in which the solution falls into local optima, and is widely applied to solving nonlinear or computationally complex problems. Figure 2 shows the overall process of GA. To determine optimal PMU placement, GA was applied as follows:

1. Generate population: The $N_{i g}$ combinations are chosen at random from a set of ${ }_{N} C_{N_{m}}$, where $N$ is the total number of buses and $N_{m}$ is the number of PMU-installed buses. Selected buses are used as input buses for LR, which estimates pseudo-measurement.

2. Evaluate average TVE: Using $N_{m}$ voltage magnitude and phase angle data and active power and reactive power of the slack bus as inputs, $N_{u}$ voltage magnitude and phase angle of unmeasured points are estimated, where $N_{u}$ is a number of unmeasured buses. After that, convert the estimated voltage magnitude and phase angle for unmeasured points to TVE through Equation (4). The evaluation of that combination is based on the average TVE of $N_{u}$ unmeasured buses.

3. Selection: The $h_{1}$ combinations with the lowest average TVE value based on fitness values and the $h_{2}$ combinations chosen at random as lucky survivors are chosen to be the parents of the next generation.

4. Crossover: A total of $h_{1}+h_{2}$ combinations surviving from the selection process are grouped into $\left(h_{1}+h_{2}\right) / 2$ to create five combinations for each pair through the crossover process. A total of 20 pairs produce 100 combinations, resulting in 100 new combinations. The crossover process was carried out by combining the PMU placement information of each pair and then choosing the number of $N_{m}$ random extraction. 
5. Mutation: $h_{4}$ of the generated combinations are selected as mutation and one of the information (bus position that PMU is installed) is changed to a random value. After completing this process, a new generation consisting of 100 combinations is born.

6. Return to the "Evaluate average TVE" process. These processes are repeated until the $N_{i g}$ th generation. The information (PMU position) of the gene that had the best value since the $N_{i g}$ th generation is selected as the optimal PMU.

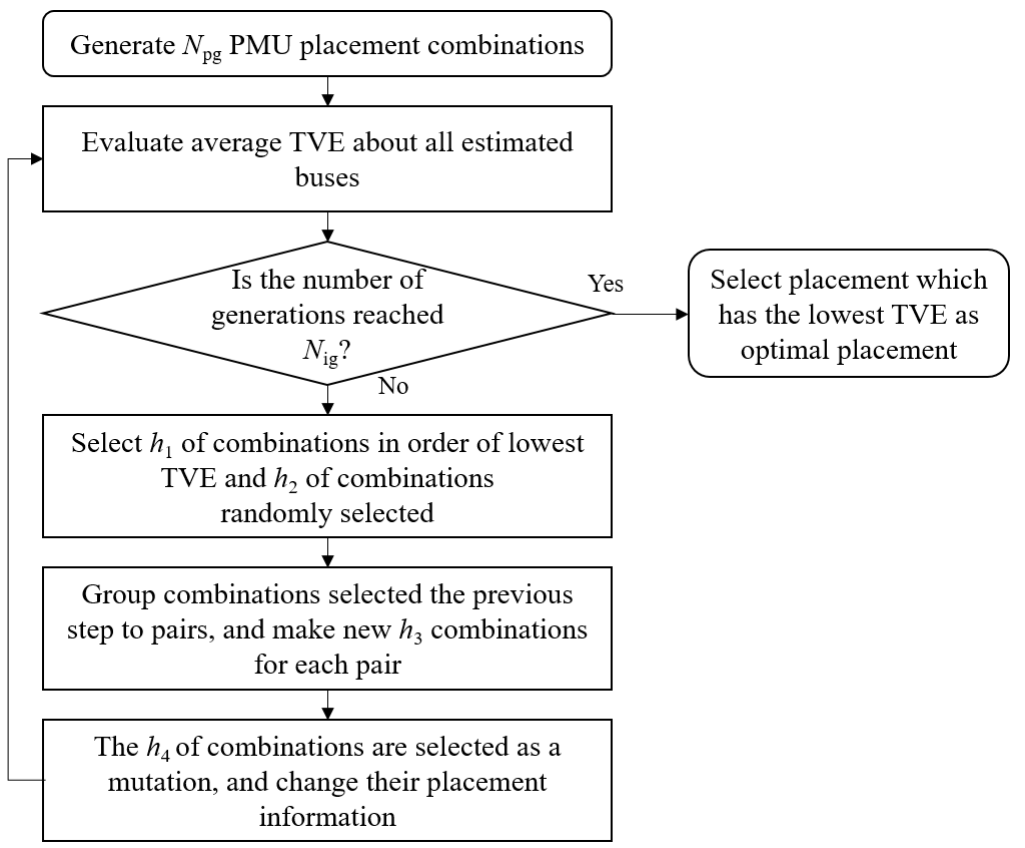

Figure 2. Flowchart of GA.

The parameters of GA are considered as shown in Table 1.

Table 1. Parameters of GA.

\begin{tabular}{ccc}
\hline Parameter & Description & Values \\
\hline$N_{p g}$ & Population sizes & 100 \\
$h_{1}$ & Best samples & $20 \%$ \\
$h_{2}$ & Lucky samples & $20 \%$ \\
$h_{3}$ & Number of children & 5 \\
$h_{4}$ & Chance of mutation & $20 \%$ \\
$N_{i g}$ & Final generation & 20 \\
\hline
\end{tabular}

\subsection{Particle Swarm Optimization}

Particle swarm optimization (PSO) is an optimization technique implemented by mimicking the collective behavioral characteristics of organisms such as birds and fish. The value of the candidate solution expressed as the position of the particle is found through iterative computation to find the optimal solution of the objective function, and the position of the candidate solution is updated through a simple equation as shown below (Equations (9) and (10)).

$$
\begin{gathered}
\mathbf{x}_{i}(t+1)=\mathbf{x}_{i}(t)+\mathbf{v}_{i}(t+1), \\
\mathbf{v}_{i}(t+1)=w \mathbf{v}_{i}(t)+c_{1}\left(\mathbf{p}_{i}-\mathbf{x}_{i}(t)\right) R_{1}+c_{2}\left(\mathbf{g}-\mathbf{x}_{i}(t)\right) R_{2},
\end{gathered}
$$

where $\mathbf{x}_{i}$ is the position vector of the $i$ th particle, and the new position $\mathbf{x}_{i}(t+1)$ is updated reflecting the velocity at the existing position. In this paper, $\mathbf{x}_{i}$ consists of a combination of buses that placed PMUs. $\mathbf{v}_{t}$ is the velocity associated with the renewal of particles and is 
updated with reference to Table 2, where $\mathbf{p}$ is known as "personal best (pbest)". It means the coordinates of the best solution obtained so far by that specific individual, while $\mathbf{g}$ is the "global best (gbest)", the overall best solution obtained by the swarm. The best placements of each particle are $\mathbf{p}$, and the best placement of all particles are $\mathbf{g}$ in this problem. Values of cognitive factors $\left(c_{1}\right)$ and social factors $\left(c_{2}\right)$ determine weight of $\mathbf{p}$ and $\mathbf{g}$, respectively. Inertia weight $(\mathrm{w})$ determines the contribution rate of a particle's previous velocity to its velocity at the current time step [27]. Parameters about these variables refer to [28]. In Equation (10), $R_{1}$ and $R_{2}$ are uniformly distributed random variable in the range [0,1], i.e., random numbers generated from a uniform distribution in $[0,1]$, so that both the social and the cognitive components have a stochastic influence on the velocity update rule in Equation (10).

PSO is characterized by several particles within the exploration space, used to derive optimal solutions and update their locations by considering the information of individual particles and the entire group at the same time. A detailed description of this technique can be found in $[27,29]$. Figure 3 shows the PSO process. To determine the optimal PMU placement using PSO, a PSO algorithm was applied as follows:

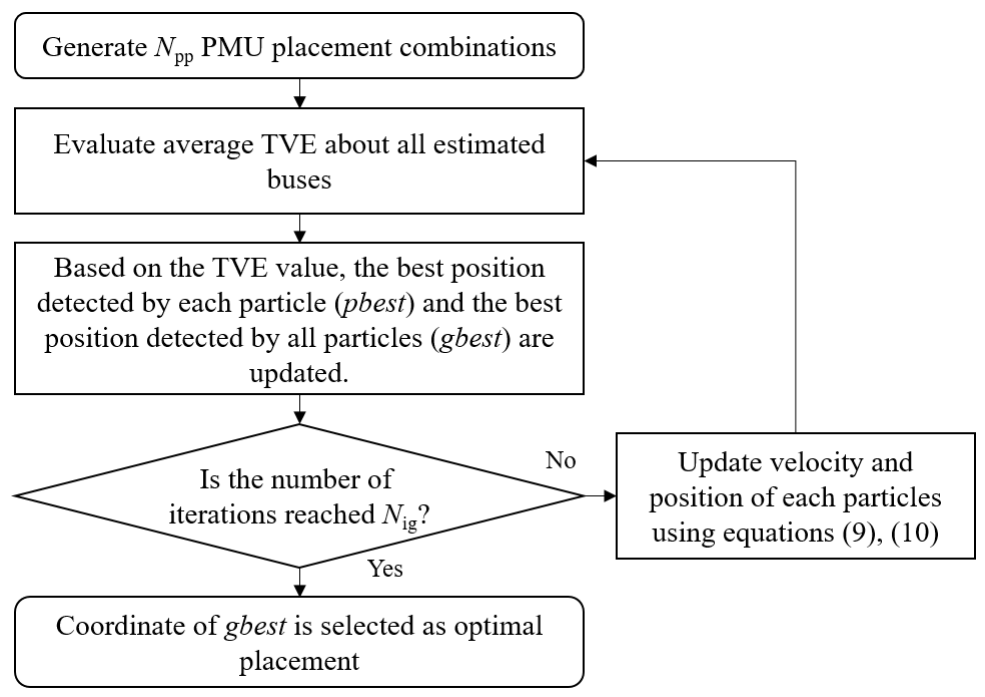

Figure 3. Flowchart of PSO.

1. Initialization: $N_{p p}$ combinations of PMU placement were generated. The combinations were generated at random from the combination set ${ }_{N} \mathrm{C}_{N_{m}}$, where $N$ is the system size (total bus number) and $N_{m}$ is the number of PMU-installed buses. The combinations that occurred were applied as the PMU installation point in the simulation.

2. Evaluate the fitness value: The average TVE of $N_{u}$ unmeasured buses becomes the evaluation of that combination. The calculation of fitness values was carried out with Equation (4), such as the approach used with the GA described earlier.

3. Update gbest and pbest: Based on the fitness value, the position with the lowest average TVE value for an individual particle (pbest) and the position with the lowest TVE value for all particles (gbest) are updated.

4. If the number of iterations is reaches $N_{i g}$, the process moves on to step 6, otherwise, it moves on to step 5 .

5. Velocity and position were updated: the velocity equation reflecting inertial, social, and cognitive factors was used to update the position of each particle. The parameter information is shown in Table 2. The position of each updated particle moves back to step 2, and the process is repeated.

6. After all iterations are terminated, the coordinate of gbest is selected as the optimal placement.

The parameters of PSO are considered as shown in Table 2. 
Table 2. Parameters of PSO.

\begin{tabular}{ccc}
\hline Parameter & Description & Values \\
\hline$c_{1}$ & Cognitive factor & 2.0 \\
$c_{2}$ & Social factor & 2.0 \\
$w$ & Inertia weight & 0.9 \\
$N_{p p}$ & Particles & 100 \\
$N_{i p}$ & Max iteration & 20 \\
$d$ & Dimension & $N_{m}$ \\
\hline
\end{tabular}

\section{Simulation Result}

\subsection{Optimal PMU Placement}

The effectiveness of the metaheuristic algorithm for optimal PMU placement was tested. The simulation was conducted assuming that there were three, four, and five PMUs installed in each of the two test feeder cases. The results obtained from the metaheuristic algorithm were compared to the RS algorithm. RS was first proposed by Brooks in 1958 [30]. RS belongs to a family of numerical optimization methods that do not require optimization of the slope of the problem, so they can be used for noncontinuous or differentiable functions. The RS method does not place any constraints on the search process, so it is easy to search for uninformed problems. In this study, the average value for each search was obtained from a total of 10,000 trials. For example, in the 100th search (first generation), the minimum value obtained by RS occurred 10,000 times in total, and the average minimum value was obtained, which was repeated until 100, 200, ..., and 2000 searches had been completed. All techniques were developed in the Python environment.

\subsubsection{Case 1-IEEE 33-Bus System}

Figure 4 shows the single-line circuit diagram of the IEEE 33-bus system. All data for this system were configured with reference to [31].

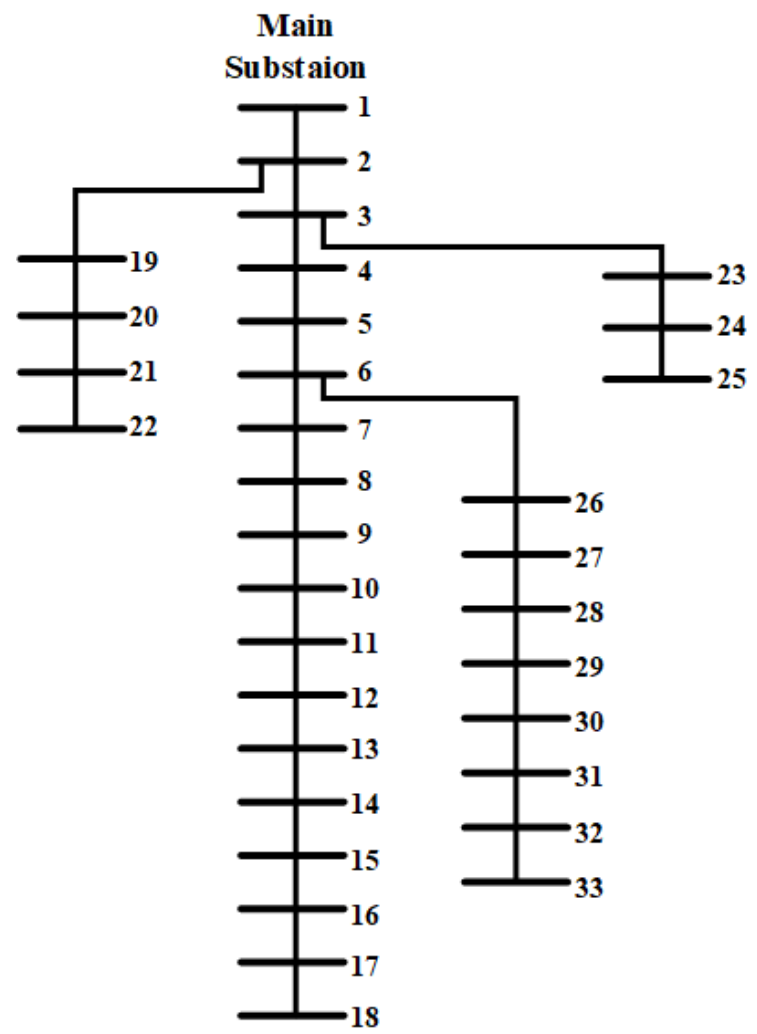

Figure 4. IEEE 33-bus system. 
In Table 3, 'Rank' represents the rank that the algorithm found among all possible combinations of the case, and 'top \%' is an indicator of the top percentage of the combination, 'Exploration' represents the minimum number of combinatorial searches taken to find that rank, and 'top' indicates that the placement combination corresponds to the best placement. IB is the position of buses derived by each algorithm. Comparing the algorithm results with three, four, and five PMUs installed, metaheuristic techniques always found a better combination than random exploration. In addition, although it explored far fewer combinations than BF, it selected the top-level combination as the best location. In this task, GA and PSO explore every possible combination based on 2000 searches.

Figures 5-7 show the ranks of the PMU placements found by each technique, as the number of explorations increases. The first iteration of the metaheuristic technique, similar to random exploration, did not appear to be superior to RS because 100 random combinations were selected. However, from the second iteration onward, the exploration was more efficient than RS.

As shown in Table 3 and Figures 5-7, metaheuristic techniques were superior to RS as the number of PMUs installed increased. In other words, the performance of the metaheuristic techniques increased with the number of combinations required for the exhaustive search.

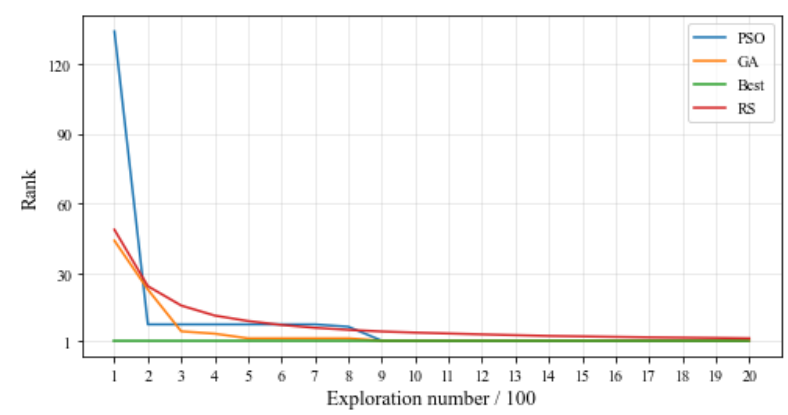

Figure 5. PMU placement ranking based on number of exploration: 33-bus system, $N_{m}=3$.

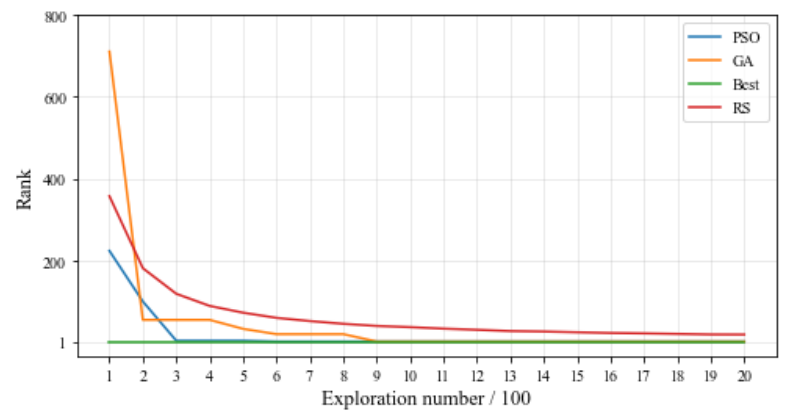

Figure 6. PMU placement ranking based on number of exploration: 33-bus system, $N_{m}=4$.

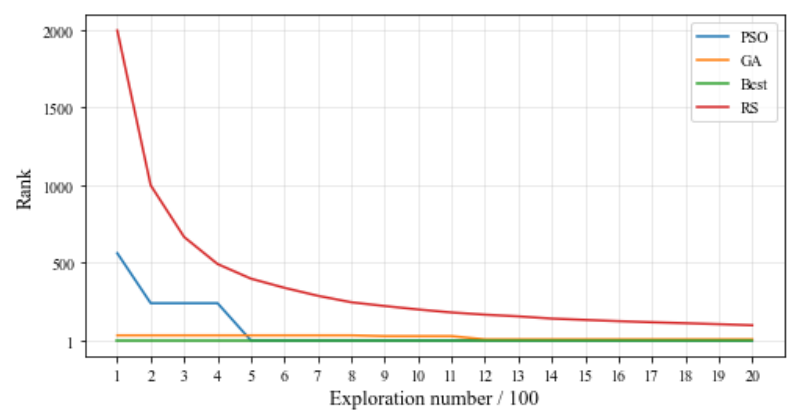

Figure 7. PMU placement ranking based on number of exploration: 33-bus system, $N_{m}=5$. 
Table 3. PMU placement results-33-bus system.

\begin{tabular}{ccccc}
\hline Method & $\boldsymbol{N}_{m}$ & Rank (Top \%) & Exploration & IB \\
\hline \multirow{3}{*}{ GA } & 3 & 1 (top) & 900 & $9,16,31$ \\
& 4 & 1 (top) & 900 & $9,16,24,31$ \\
& 5 & $8(0.004)$ & 1200 & $6,10,16,24,31$ \\
\multirow{2}{*}{ PSO } & 3 & 1 (top) & 900 & $9,16,31$ \\
& 4 & 1 (top) & 600 & $9,16,24,31$ \\
& 5 & 1 (top) & 900 & $10,16,24,26,31$ \\
RS & 3 & $3(0.06)$ & 2000 & $10,16,31$ \\
& 4 & $18(0.05)$ & 2000 & $9,16,24,32$ \\
& 5 & $101(0.05)$ & 2000 & $6,10,17,23,31$ \\
BF & 3 & 1 (top) & 4960 & $9,16,31$ \\
& 4 & 1 (top) & 35,960 & $9,16,24,31$ \\
& 5 & 1 (top) & 201,376 & $10,16,24,26,31$ \\
\hline
\end{tabular}

$N_{m}$ : Number of Installed PMU, IB: Identified Buses, RS: Random Search, BF: Brute Force.

\subsubsection{Case 2-IEEE 69-Bus System}

Figure 8 shows a single line circuit diagram of the 69-bus system. All of the data of this system were configured with reference to [32]. The results obtained through each algorithm are shown in Table 4. Regardless of the number of PMUs installed, metaheuristic techniques were superior to RS, as in the previous case.

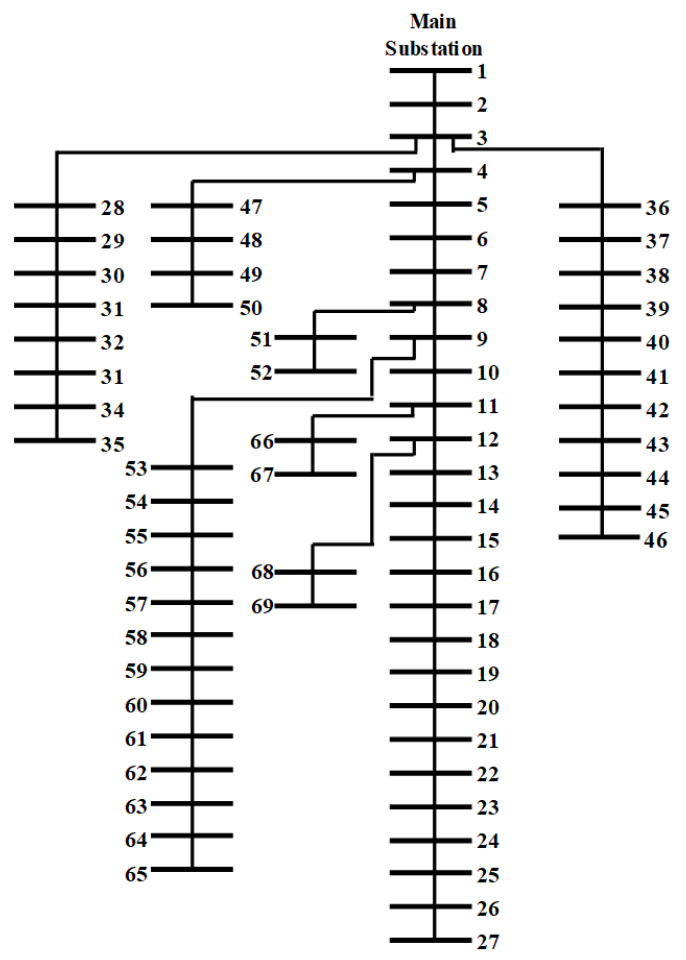

Figure 8. IEEE 69-bus system.

Figures 9-11 show the ranks of the PMU placements derived by each technique, as the number of explorations increased in the 69-bus system. At the beginning of the exploration, superiority over random exploration was difficult, but as the exploration progressed, OP was found more effectively. Optimal PMU placement via metaheuristic techniques tended to show better performance as the number of combinations required for exploration increased. In the case of five installed PMUs, the total number of combinations corresponding to an exhaustive search was ${ }_{68} C_{5}, 10,424,128$; however, only 2000 combinations $(0.019 \%)$ reached the top $0.0036 \%$ rank. 
Table 4. PMU placement results-69-bus system.

\begin{tabular}{ccccc}
\hline Method & $\boldsymbol{N}_{m}$ & Rank (Top \%) & Exploration & IB \\
\hline \multirow{3}{*}{ GA } & 3 & $4(0.008)$ & 1800 & $20,60,67$ \\
& 4 & $38(0.005)$ & 2000 & $20,43,62,67$ \\
& 5 & $680(0.007)$ & 1000 & $20,43,62,63,66$ \\
\multirow{2}{*}{ PSO } & 3 & 1 (top) & 1900 & $10,20,60$ \\
& 4 & $19(0.002)$ & 1300 & $20,44,60,67$ \\
& 5 & $371(0.004)$ & 1600 & $10,20,46,63,64$ \\
RS & 3 & $24(0.048)$ & 2000 & $10,24,60$ \\
& 4 & $402(0.049)$ & 2000 & $11,19,60,64$ \\
& 5 & $5077(0.049)$ & 2000 & $6,13,20,43,60$ \\
BF & 3 & 1 (top) & 50,116 & $10,20,60$ \\
& 4 & 1 (top) & 814,385 & $10,20,43,60$ \\
& 5 & 1 (top) & $10,424,128$ & $10,20,43,60,65$ \\
\hline
\end{tabular}

$N_{m}$ : Number of Installed PMU, IB: Identified Buses, RS: Random Search, BF: Brute Force.

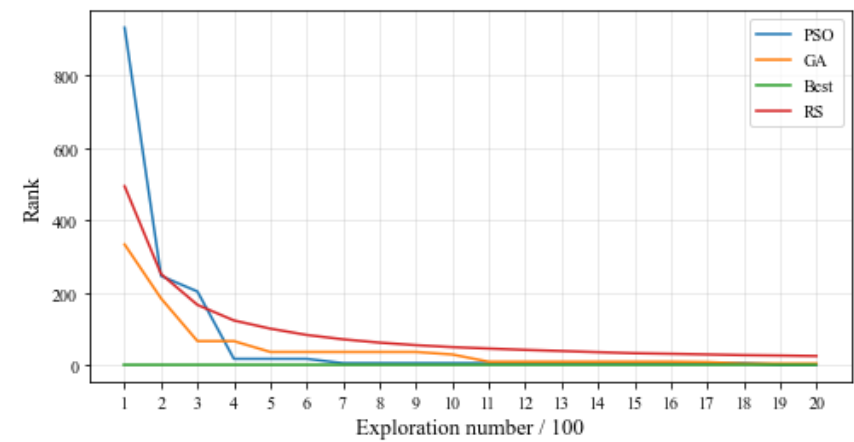

Figure 9. PMU placement ranking based on number of exploration-69-bus system, $N_{m}=3$.

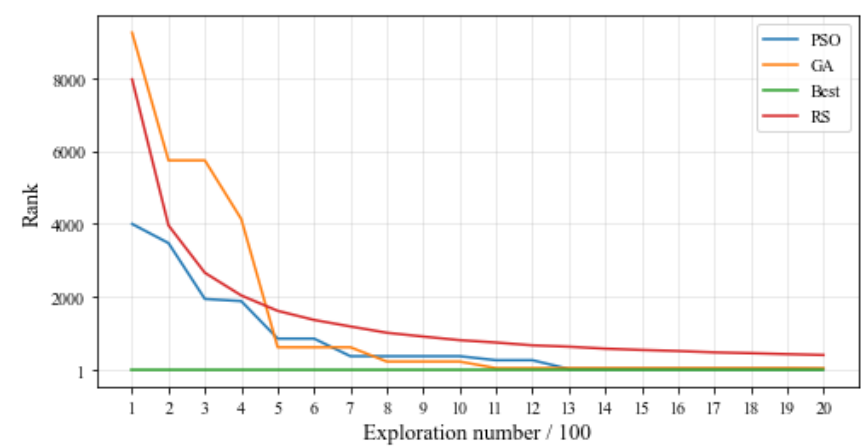

Figure 10. PMU placement ranking based on number of exploration-69-bus system, $N_{m}=4$.

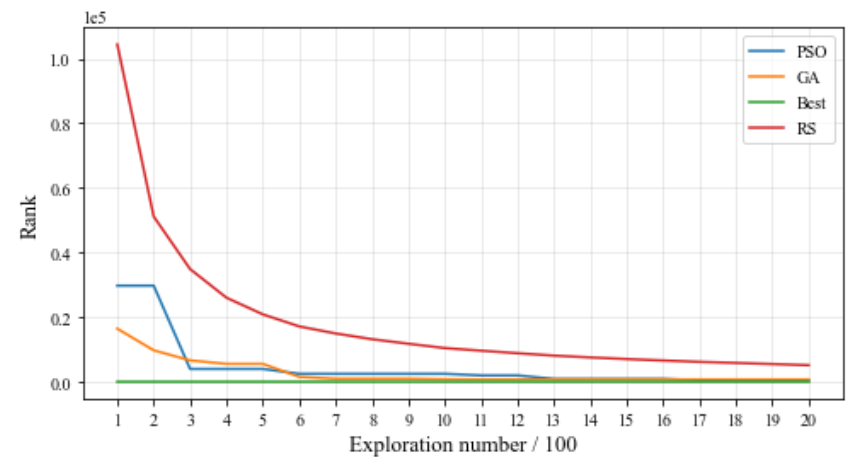

Figure 11. PMU placement ranking based on number of exploration-69-bus system, $N_{m}=5$. 


\subsection{State Estimation}

To verify the effect of OP on state estimation, a weighted least squares algorithm [33] was employed. Cases of pseudo-measurements generated from OP, and worst placement (WP) and pseudo-measurements generated through LPs, were compared. In this paper, the measurement uncertainty of PMU and each pseudo-measurement was considered as follows:

1. Measurement uncertainty of PMU: $1 \%$ for voltage magnitude, $0.573^{\circ}$ for phase angle [34].

2. Pseudo-measurements based on OP and WP: Uncertainty in values estimated by LR.

3. Pseudo-measurements based on LP: $50 \%$ for active/reactive power.

State estimation was performed for the IEEE 33- and 69-bus systems. The state estimation results showed remarkable improvement when the pseudo-measurements modeled by supervised learning techniques (based on OP) were used, regardless of the number of PMUs installed.

\subsubsection{Case 1-IEEE 33-Bus System}

The results of the state estimation conducted for the IEEE 33-bus system are as follows: Table 5 shows the voltage magnitude and angle errors for the 33-bus system. Pseudomeasurements generated by supervised learning techniques (OP, WP) were compared to those based on LPs.

Table 5. State estimation error-33-bus system.

\begin{tabular}{cccccccc}
\hline \multirow{2}{*}{$N_{m}$} & \multirow{2}{*}{ Indicator } & \multicolumn{3}{c}{ Magnitude $\left(\times \mathbf{1 0}^{-\mathbf{6}} \mathbf{p . u}\right)$} & \multicolumn{3}{c}{ Angle $\left(\times \mathbf{1 0}^{-\mathbf{3}}\right.$ Degree $)$} \\
\cline { 3 - 8 } & & OP & LP & WP & OP & LP & WP \\
\hline \multirow{3}{*}{3} & Avg & 71.294 & 175.094 & $15,556.095$ & 1.977 & 15.514 & 2074.534 \\
& Max & 621.567 & 780.11 & $66,611.141$ & 14.991 & 84.133 & 8413.159 \\
& Min & 0 & 0 & 0 & 0 & 0 & 0 \\
4 & Avg & 55.443 & 155.081 & $16,795.932$ & 1.096 & 14.629 & 1974.514 \\
& Max & 349.5 & 755.405 & $67,255.438$ & 3.3869 & 49.88 & 8398.775 \\
& Min & 0 & 0 & 0 & 0 & 0 & 0 \\
5 & Avg & 41.741 & 147.8 & $17,136.878$ & 0.41 & 9.2 & 1578.64 \\
& Max & 409.756 & 762.125 & $59,708.604$ & 2.993 & 35.865 & 7263.886 \\
& Min & 0 & 0 & 0 & 0 & 0 & 0 \\
\hline
\end{tabular}

OP: Optimal Placement, LP: Load Profile, WP: Worst Placement.

If the pseudo-measurements were generated through LR, which is based on OP, the state estimation error was lower, regardless of the number of PMUs installed, than if pseudo-measurements generated based on LP were used as state variables. However, if the PMU was installed in the WP, the estimation was higher than that of generated pseudo-measurements based on LP.

Compared to OP- and LP-based pseudo-measurements, the case of three installed PMUs showed 2.43- and 13.68-fold differences in the average voltage magnitude and phase angle, respectively, and 1.33- and 6.89-fold differences in the maximum voltage magnitude and phase angle, respectively. In the case of four PMUs being installed, 2.8- and 13.34-fold differences in the average voltage magnitude and phase angle were seen, respectively, and there were 2.16- and 14.73-fold differences in the maximum voltage magnitude and phase angle, respectively. In the case where five PMUs were installed, there were 3.54- and 22.44fold differences in the average voltage magnitude and phase angle, and 1.86- and 11.98-fold differences in the maximum voltage magnitude and phase angle error, respectively. The state estimation error with supervised learning-based pseudo-estimation in the worst case of PMU placement was much greater than when LP-based pseudo-measurements were used as state variables. In this case, as shown in Table 5, the average voltage magnitude error was $88.84,108.3$, and 115.95 times larger than the OP suggested by PSO in the cases 
where three, four, and five PMUs were installed, and the maximum error was 85.39, 89.03, and 78.34 times larger. For the phase angle error, the average error was 133.72, 134.97, and 171.59 times larger for the cases with three, four, and five PMUs installed, respectively, and the maximum error was 100, 168.38, and 202.53 times larger. The larger the number of PMUs installed overall, the greater the superiority of the optimal PMU selection.

Figures 12-14 show the state estimation error when the OP-based pseudo-measurements were used as state variables for state estimation. Figure 13 shows a graph for a PMU installed at bus No. 24 in Figure 12. Adding PMUs to bus No. 24 significantly reduced errors in bus Nos. 23 and 25, which were adjacent to bus No. 24. The tendency to reduce the error of adjacent buses was seen in all cases in which pseudo-measurements were generated based on LR techniques. Figure 14 shows a state estimation error graph for a five-PMU installation. In this case, bus Nos. 10 and 26 were added, and bus No. 9 was removed; compare to Figure 13. As a result, the error between the excluded bus and its adjacent bus No. 8 increased, and the error between the newly installed buses (Nos. 10 and 26) and their adjacent buses decreased.

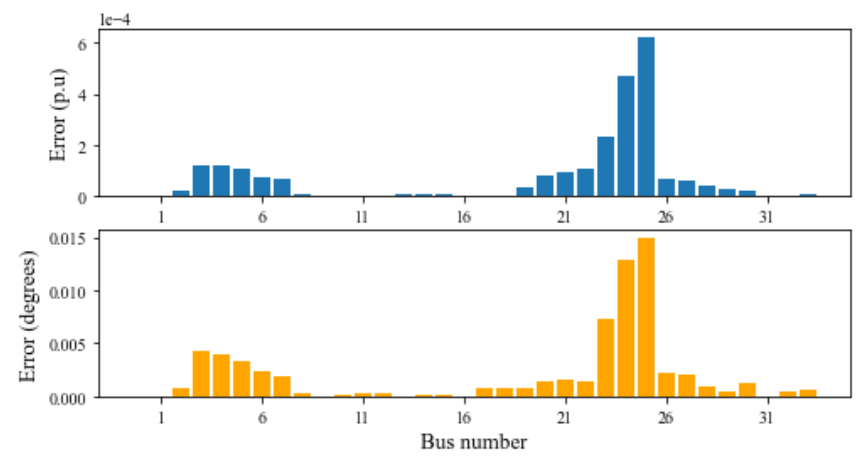

Figure 12. State estimation error (OP): 33-bus system, $N_{m}=3$.

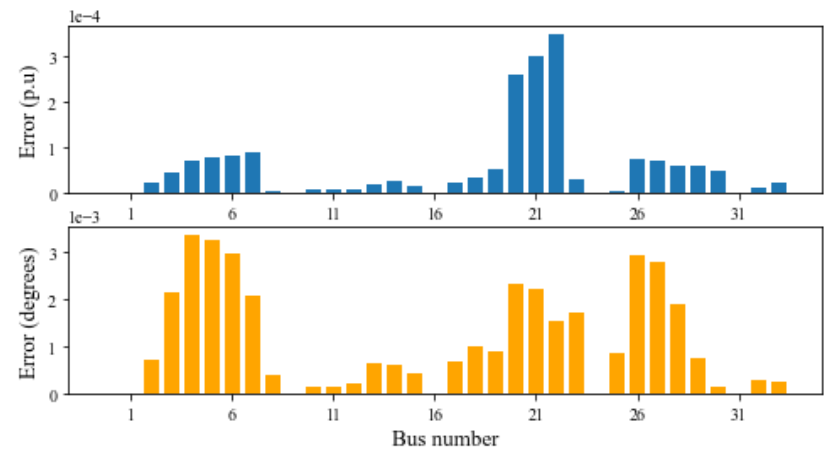

Figure 13. State estimation error (OP): 33-bus system, $N_{m}=4$.
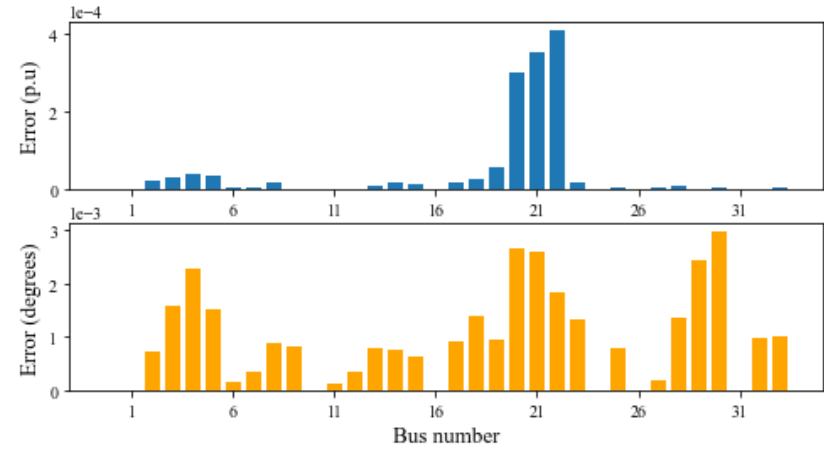

Figure 14. State estimation error (OP): 33-bus system, $N_{m}=5$. 


\subsubsection{Case 2-IEEE 69-Bus System}

The results of the state estimation conducted in the IEEE 69-bus system are as follows:

Table 6 shows the voltage magnitude and phase angle errors in the 69-bus system. A comparison was made between pseudo-measurements generated by supervised learning techniques (OP, WP) and based on LPs, which were used as state variables for state estimation.

Table 6. State estimation error-69-bus system.

\begin{tabular}{ccllllll}
\hline \multirow{2}{*}{$N_{m}$} & \multirow{2}{*}{ Indicator } & \multicolumn{3}{c}{ Magnitude $\left(\times \mathbf{1 0}^{-\mathbf{6}} \mathbf{p . u}\right)$} & \multicolumn{3}{c}{ Angle $\left(\times \mathbf{1 0}^{-\mathbf{3}}\right.$ Degree $)$} \\
\cline { 3 - 8 } & & \multicolumn{1}{c}{ OP } & \multicolumn{1}{c}{$\mathbf{L P}$} & \multicolumn{1}{c}{ WP } & \multicolumn{1}{c}{ OP } & \multicolumn{1}{c}{ LP } & WP \\
\hline \multirow{3}{*}{3} & Avg & 111.105 & 270.418 & 2156.975 & 0.999 & 13.665 & 136.253 \\
& Max & 3187.87 & 4257.55 & $13,625.349$ & 11.88 & 81.883 & 693.295 \\
& Min & 0 & 0 & 0 & 0 & 0 & 0 \\
4 & Avg & 106.465 & 204.701 & 2164.774 & 0.894 & 6.695 & 101.866 \\
& Max & 3222.997 & 4327.7 & $13,616.621$ & 12.059 & 81.866 & 691.581 \\
& Min & 0 & 0 & 0 & 0 & 0 & 0 \\
5 & Avg & 106.503 & 133.918 & 2161.948 & 0.613 & 5.098 & 136.065 \\
& Max & 3227.337 & 3816.824 & $10,186.182$ & 12.429 & 91.925 & 692.318 \\
& Min & 0 & 0 & 0 & 0 & 0 & 0 \\
\hline
\end{tabular}

OP: Optimal Placement, LP: Load Profile, WP: Worst Placement.

In this case, similar to the previous case, the pseudo-measurements generated at the OP were superior to those generated based on the LP. Compared to OP- and LP-based pseudo- measurements, in the case where three PMUs were installed, there were 2.43and 13.68-fold differences in the average voltage magnitude and phase angle, respectively, and 1.33- and 6.89-fold differences in the maximum voltage magnitude and phase angle. In the case where four PMUs were installed, there were 1.92- and 7.49-fold differences for the average voltage magnitude and phase angle, respectively, and 1.34- and 6.79-fold differences for the maximum voltage magnitude and phase angle, respectively. In the case where five PMUs were installed, there were 1.26- and 8.83-fold differences in the average voltage magnitude and phase angle, respectively, and 7.4- and 11.98-fold differences in the maximum voltage magnitude and phase angle error, respectively. On the other hand, the state estimation error when pseudo-measurements were generated based on WP was much greater than when LP-based pseudo-measurements were used as the state variables. In this case, the average voltage magnitude error was 7.97, 10.57, and 16.14 times larger than the optimal location according to PSO in the cases with three, four, and five installed PMUs, respectively, and the maximum error was 3.2, 3.15, and 2.67 times larger. For the phase angle error, the average error was 9.97 times, 15.21 times, and 26.69 times larger, and the maximum error was 8.47 times, 8.45 times, and 7.53 times larger. Overall, the larger the number of PMUs installed, the greater the superiority of the optimal PMU selection.

Figures 15-17 show the state estimation error when OP-based pseudo-measurements were used as state variables in the 69-bus system. Figure 16 shows the case of a PMU installed in addition to bus No. 24 in Figure 15. Adding PMUs to bus No. 24 also significantly reduced the errors for buses No. 23 and 25, which are adjacent to bus No. 24 . The tendency to also reduce the error for adjacent buses was seen in all cases in which pseudo-measurements were generated based on LR techniques. The phase angle error of the excluded bus and its adjacent bus increased, while the error of the added bus and its adjacent bus decreased. Figure 17 shows the state estimation error when buses No. 44, 60, and 67 were excluded from Figure 16 and buses No. 10, 46, 63, and 64 were added. In this case, as well, the overall error for the excluded bus and its adjacent bus increased, while the error for the added bus and its adjacent bus decreased. 


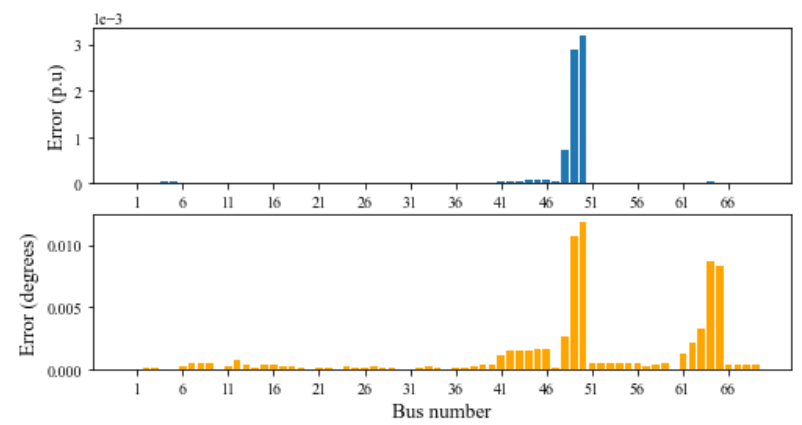

Figure 15. State estimation error (OP) -69-bus system, $N_{m}=3$.

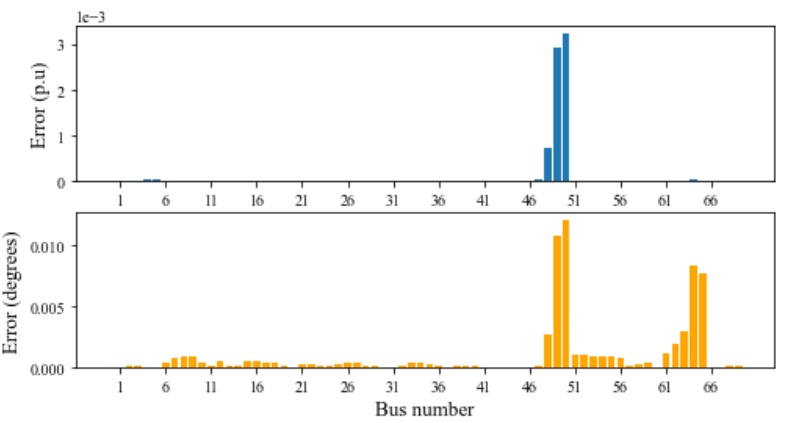

Figure 16. State estimation error (OP) -69-bus system, $N_{m}=4$.

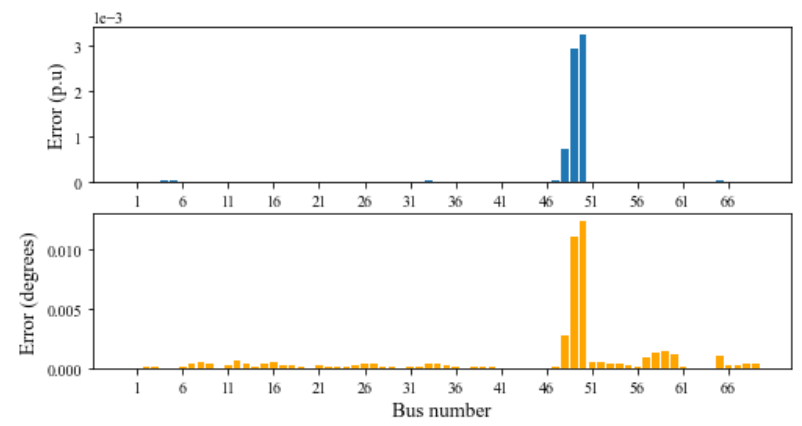

Figure 17. State estimation error (OP) -69-bus system, $N_{m}=5$.

\section{Conclusions}

This paper presents a framework of optimal PMU placement for pseudo-measurements modeling based on supervised learning. Two metaheuristic algorithms, GA and PSO, were used to determine the optimal PMU placement in an attempt to generate more accurate pseudo-measurements. Subsequently, the pseudo-measurement models estimated by supervised learning techniques were used as state variables for state estimation. GA and PSO techniques provided more optimal PMU placement for supervised learning-based pseudo-measurement modeling than RS. The proposed approaches were simulated in IEEE 33- and 69-bus systems. These techniques became increasingly more effective as the number of buses in the system increased or the number of PMUs installed became closer to half the number of buses. In other words, as the total number of combinations in the exhaustive search increased, the algorithms became more effective. Within this framework, supervised learning-based pseudo-measurement modeling and the optimal placement for improving its performance can be considered together. In addition, the framework is designed generally, so it is scalable when applying metaheuristic algorithms or supervised learning techniques not covered in this paper or newly developed. However, in this paper, communication delay of PMU, renewable power sources, and the number of installed PMUs are not considered, so additional verification and research remain to be performed in future works. 
Author Contributions: Methodology, software, and validation, K.-Y.L.; writing—review and editing, K.-Y.L. and J.-S.P.; conceptualization and writing-original draft preparation, Y.-S.K. All authors have read and agreed to the published version of the manuscript.

Funding: This research was funded by Energy Technology Evaluation and Planning(KETEP). (Grant number: 20204010600340) in 2021.

Institutional Review Board Statement: Not applicable.

Informed Consent Statement: Not applicable.

Data Availability Statement: Not applicable.

Acknowledgments: This work was supported by the Korea Institute of Energy Technology Evaluation and Planning (KETEP) grant funded by the Korea government (MOTIE) (20194310100030, Development of Demonstration Zone for New Electricity Service Model). This work was supported by the Korea Institute of Energy Technology Evaluation and Planning (KETEP) and the Ministry of Trade, Industry \& Energy (MOTIE) of the Republic of Korea (No. 20204010600340).

Conflicts of Interest: The authors declare no conflict of interest.

\section{References}

1. Etherden, N.; Johansson, A.K.; Ysberg, U.; Kvamme, K.; Pampliega, D.; Dryden, C. Enhanced LV supervision by combining data from meters, secondary substation measurements and medium voltage supervisory control and data acquisition. CIRED-Open Access Proc. J. 2017, 2017, 1089-1093. [CrossRef]

2. Rahman, M.A.; Jakaria, A.H.M.; Al-Shaer, E. Formal Analysis for Dependable Supervisory Control and Data Acquisition in Smart Grids. In Proceedings of the 2016 46th Annual IEEE/IFIP International Conference on Dependable Systems and Networks (DSN), Toulouse, France, 28 June-1 July 2016; pp. 263-274. [CrossRef]

3. Liao, J.; He, C. Wide-area monitoring protection and control of future power system networks. In Proceedings of the 2014 IEEE Workshop on Advanced Research and Technology in Industry Applications (WARTIA), Ottawa, ON, Canada, 29-30 September 2014; pp. 903-905. [CrossRef]

4. Chaudhuri, N.R. Wide-Area Monitoring and Control of Smart Energy Cyber-Physical Systems (CPS). Smart Cities Found. Princ. Appl. 2017, 155-180. [CrossRef]

5. Baran, M.; Zhu, J.; Kelley, A. Meter placement for real-time monitoring of distribution feeders. IEEE Trans. Power Syst. 1996, 11, 332-337. [CrossRef]

6. Liu, H.; Yu, D.; Chiang, H.D. A heuristic meter placement method for load estimation. IEEE Trans. Power Syst. 2002, 17, 913-917. [CrossRef]

7. Singh, R.; Pal, B.C.; Jabr, R.A.; Vinter, R.B. Meter placement for distribution system state estimation: An ordinal optimization approach. IEEE Trans. Power Syst. 2011, 26, 2328-2335. [CrossRef]

8. Celli, G.; Pegoraro, P.A.; Pilo, F.; Pisano, G.; Sulis, S. DMS Cyber-Physical Simulation for Assessing the Impact of State Estimation and Communication Media in Smart Grid Operation. IEEE Trans. Power Syst. 2014, 29, 2436-2446. [CrossRef]

9. El-Zonkoly, A. Optimal meter placement using genetic algorithm to maintain network observability. Expert Syst. Appl. 2006, 31, 193-198. [CrossRef]

10. Kumar, A.; Das, B.; Sharma, J. Genetic algorithm-based meter placement for static estimation of harmonic sources. IEEE Trans. Power Deliv. 2005, 20, 1088-1096. [CrossRef]

11. Chakrabarti, S.; Venayagamoorthy, G.K.; Kyriakides, E. PMU placement for power system observability using binary particle swarm optimization. In Proceedings of the 2008 Australasian Universities Power Engineering Conference, Sydney, Australia, 14-17 December 2008; pp. 1-5.

12. Watitwa, J.; Awodele, K. A Review on Active Distribution System State Estimation. In Proceedings of the 2019 Southern African Universities Power Engineering Conference/Robotics and Mechatronics/Pattern Recognition Association of South Africa (SAUPEC/RobMech/PRASA), Bloemfontein, South Africa, 28-30 January 2019; pp. 726-731. [CrossRef]

13. Manitsas, E.; Singh, R.; Pal, B.C.; Strbac, G. Distribution System State Estimation Using an Artificial Neural Network Approach for Pseudo Measurement Modeling. IEEE Trans. Power Syst. 2012, 27, 1888-1896. [CrossRef]

14. DAS, B. Rule Based Algorithm for Meter Placement and ANN Based Bus Voltage Estimation in Radial Power Distribution System. Electr. Power Compon. Syst. 2005, 33, 449-462. [CrossRef]

15. Hong, G.; Kim, Y. Supervised Learning Approach for State Estimation of Unmeasured Points of Distribution Network. IEEE Access 2020, 8, 113918-113931. [CrossRef]

16. Abdel-Majeed, C.A.; Kattmann, S.T.; Saur, R. Usage of Artificial Neural Networks for pseudo measurement modeling in low voltage distribution systems. In Proceedings of the 2014 IEEE PES General Meeting I Conference \& Exposition, National Harbor, MD, USA, 27-31 July 2014; pp. 1-5.

17. Cao, Z.; Wang, Y.; Chu, C.C.; Gadh, R. Robust pseudo-measurement modeling for three-phase distribution systems state estimation. Electr. Power Syst. Res. 2020, 180, 106138. [CrossRef] 
18. Adinolfi, F.; D'Agostino, F.; Morini, A.; Saviozzi, M.; Silvestro, F. Pseudo-measurements modeling using neural network and Fourier decomposition for distribution state estimation. In Proceedings of the IEEE PES Innovative Smart Grid Technologies Europe, Istanbul, Turkey, 12-15 October 2014; pp. 1-6.

19. Qiang, Q.; Sun, G.; Xu, W.; Yan, M.; Wei, Z.; Zang, H. Distribution System State Estimation Based on Pseudo Measurement Modeling Using Convolutional Neural Network. In Proceedings of the 2018 China International Conference on Electricity Distribution (CICED), Tianjin, China, 17-19 September 2018; pp. 2416-2420. [CrossRef]

20. Wang, Y.; Xia, M.; Chen, Q.; Chen, F.; Yang, X.; Han, F. Fast state estimation of power system based on extreme learning machine pseudo-measurement modeling. In Proceedings of the 2019 IEEE Innovative Smart Grid Technologies-Asia (ISGT Asia), Chengdu, China, 21-24 May 2019; pp. 1236-1241.

21. Bianchi, L.; Dorigo, M.; Gambardella, L.M.; Gutjahr, W.J. A survey on metaheuristics for stochastic combinatorial optimization. Nat. Comput. 2009, 8, 239-287. [CrossRef]

22. Dokeroglu, T.; Sevinc, E.; Kucukyilmaz, T.; Cosar, A. A survey on new generation metaheuristic algorithms. Comput. Ind. Eng. 2019, 137, 106040. [CrossRef]

23. Zabinsky, Z.B. Stochastic Adaptive Search for Global Optimization; Springer Science \& Business Media: Berlin/Heidelberg, Germany, 2013; Volume 72.

24. Groner, L. Learning JavaScript Data Structures and Algorithms; Packt Publishing Ltd.: Birmingham, UK, 2016.

25. IEEE Std C37.118.1-2011 (Revision of IEEE Std C37.118-2005). IEEE Standard for Synchrophasor Measurements for Power Systems. pp. 1-61. Available online: https://ieeexplore.ieee.org/document/6111219 (accessed on 28 December 2011). [CrossRef]

26. Mitchell, M. An Introduction to Genetic Algorithms; MIT Press: Cambridge, MA, USA, 1998.

27. Shi, Y.; Eberhart, R. A modified particle swarm optimizer. In Proceedings of the 1998 IEEE International Conference on Evolutionary Computation Proceedings. IEEE World Congress on Computational Intelligence (Cat. No.98TH8360), Anchorage, AK, USA, 4-9 May 1998; pp. 69-73. [CrossRef]

28. Kennedy, J.; Eberhart, R. Particle swarm optimization. In Proceedings of the ICNN'95-International Conference on Neural Networks, Perth, Australia, 27 November-1 December 1995; Volume 4, pp. 1942-1948. [CrossRef]

29. Marini, F.; Walczak, B. Particle swarm optimization (PSO). A tutorial. Chemometr. Intell. Lab. Syst. 2015, 149, 153-165. [CrossRef]

30. Brooks, S.H. A discussion of random methods for seeking maxima. Oper. Res. 1958, 6, 244-251. [CrossRef]

31. Baran, M.; Wu, F. Network reconfiguration in distribution systems for loss reduction and load balancing. IEEE Trans. Power Deliv. 1989, 4, 1401-1407. [CrossRef]

32. Savier, J.S.; Das, D. Impact of Network Reconfiguration on Loss Allocation of Radial Distribution Systems. IEEE Trans. Power Deliv. 2007, 22, 2473-2480. [CrossRef]

33. Abur, A.; Exposito, A.G. Power System State Estimation: Theory and Implementation; CRC Press: Boca Raton, FL, USA, 2004.

34. Narendra, K.; Gurusinghe, D.R.; Rajapakse, A.D. Dynamic performance evaluation and testing of phasor measurement unit (PMU) as per IEEE C37. 118.1 Standard. In Proceedings of the Doble Client Committee Meetings International Protection Testing Users Group, Chicago, IL, USA, 1 October 2012; Volume 10. 